\title{
PREVALENCE AND ANTIBIOGRAM OF EXTENDED SPECTRUM BETA- LACTAMASE PRODUCING ESCHERICHIA COLI
}

Mohd. Rashid, Syamal Modi, Indu Shukla, Yogesh Chander

1. Assistant Professor. Department of Microbiology, SMS \& R, Sharda University, Greater Noida, India.

2. Tutor. Department of Microbiology, Patna Medical College \& Hospital, Patna, India.

3. Professor \& Head, Department of Microbiology, J.N. Medical College, AMU, Aligarh, India.

4. Professor \& Head. Department of Microbiology, SMS \& R, Sharda University, Greater Noida, India.

\section{CORRESPONDING AUTHOR:}

Dr. Mohd. Rashid, Department of Microbiology,

SMS \& R, Sharda University,

Greater Noida, India.

E-mail: rashidmohddr79@yahoo.co.in

ABSTRACT: BACKGROUND: Beta lactams are the most extensively used group of antimicrobials, however growing resistance to these invaluable drugs mediated by extended spectrum beta-lactamase (ESBL) enzymes is a major concern. The frequency of ESBL producing strains among clinical isolates has been steadily increasing over the past few years that has generated a major problem in clinical therapeutics. OBJECTIVES: Our aim was to determine the prevalence of ESBL producing Escherichia coli, study their antibiogram and to evaluate the association between ESBL production and antibiotic resistance in Escherichia coli. SETTINGS AND DESIGN: This prospective study was conducted in the Department of Microbiology, Jawaharlal Nehru Medical College, Aligarh Muslim University, Aligarh. STATISTICAL ANALYSIS USED: Chi-square test was used to analyze the data statistically. Probability values less than 0.05 were considered significant. MATERIALS AND METHODS: Two hundred and eighty six consecutive, non-repeated isolates of Escherichia coli obtained from pus, urine, blood, stool, throat swab, cervical swab, sputum, CSF and conjunctival swab samples received in our bacteriology laboratory were analyzed in this study. These bacterial isolates were identified and tested for antibiotic sensitivity by standard microbiological procedures. Subsequently, they were screened and then phenotypically confirmed for ESBL production by phenotypic confirmatory disk diffusion test (PCDDT). RESULTS: Out of 286 isolates of Escherichia coli screened for ESBL production, 65.03\% $(n=186)$ were detected to be positive using either ceftazidime or cefotaxime. In the screen positives, $91.94 \%(n=171)$ were phenotypically confirmed ESBL producers by PCDDT method. The overall prevalence of ESBL producing Escherichia coli was $59.79 \%(\mathrm{n}=171 / 286)$ with $87.72 \%$ obtained from in-patients and $12.28 \%$ from out-patients. Majority of ESBL producing Escherichia coli were recovered from stool (73.33\%), followed by pus (62.62\%), urine (61.80\%) and blood $(60.61 \%)$ samples. The antibiogram revealed no resistance to imipenem, while the highest resistance rate was detected against cefoperazone, amoxicillin/clavulanic acid, ampicillin, cefotaxime, aztreonam, cefoxitin, piperacillin, ceftazidime, and ceftriaxone. CONCLUSIONS: ESBL producing strains of Escherichia coli show extremely wide spectrum of antibiotic resistance including resistance to penicillins, cephalosporins, aminoglycosides, and fluoroquinolones. This leads to significant implications in the management of patients. Advanced drug resistance surveillance and determination of molecular characteristics of ESBL isolates are necessary to formulate antibiotic prescription policies, so as to ensure appropriate and judicious use of the available antimicrobial drugs. 
KEY WORDS: Antibiogram, Escherichia coli, Extended spectrum beta lactamase, Penicillin binding proteins, Phenotypic confirmatory disk diffusion test.

INTRODUCTION: The spectrum of drug resistance in general and multidrug resistance in particular, looms large ahead as never before. The war waged between microorganisms and antimicrobials continues to sparkle unabated with each partner developing new weaponry and seeking novel ways of struggle. Beta lactam ( $\beta$-lactam) group of antibiotics are the workhorse antimicrobial agents in routine management of patients, but the rising prevalence of extended spectrum $\beta$ - lactamase (ESBL) mediated resistance is giving clinicians a tough time to advise appropriate antimicrobials to their patients as the therapeutic options are limited.

Resistance to $\beta$ - lactam antibiotics: Resistance to penicillins and other $\beta$ - lactams in Gramnegative bacilli (prototype Escherichia coli) can occur by four general mechanisms.

1. Inactivation of antibiotic by $\beta$ - lactamase.

2. Modification of target penicillin binding proteins (PBPs).

3. Impaired penetration of drug to target PBPs.

4. Efflux mechanism.

Of these, antibiotic inactivation by $\beta$ - lactamase production is the most common mechanism of resistance.

\section{$\beta$-lactamases:}

$\beta$-lactamases are bacterial enzymes that catalyze the hydrolysis of the $\beta$-lactam ring to yield inactive products. In Gram-negative bacilli, $\beta$-lactamase enzymes remain in the periplasmic space, where they attack the $\beta$-lactam before it can reach the PBPs. Since the development and introduction of $\beta$-lactam antibiotics into clinical use, the $\beta$-lactamases have evolved with them. Today, hundreds of different $\beta$-lactamases have been identified. Genes encoding $\beta$-lactamase enzymes are found on the chromosome as well as on plasmids. Plasmid-mediated $\beta$-lactamase genes are the most common ones as they can be transferred to other Gram-negative bacteria by conjugation. Many Gram-negative bacilli possess naturally occurring chromosomally-mediated $\beta$-lactamases. They may have some physiological role in prostaglandin assembly or have evolved to defend bacteria against $\beta$-lactams produced by environmental fungi. [1] Today, it is known that the major factor causing $\beta$-lactamase production by pathogens is the widespread, injudicious and irrational clinical use of $\beta$ - lactams.

Definition of ESBL: By definition, ESBLs are molecular class A or D $\beta$-lactamases, which (i) are able to hydrolyze oxyimino cephalosporins at a rate equal to or higher than $10 \%$ of that for benzylpenicillin, (ii) have an active-site serine, and (iii) generally are inhibited by $\beta$-lactamase inhibitors such as clavulanic acid, sulbactam, or tazobactam. ${ }^{[1,2]}$ Typically, they are derived from genes for TEM-1, TEM-2, or SHV-1 by mutations that alter the amino acid configuration around the active site of these $\beta$-lactamases. This extends the spectrum of $\beta$-lactam antibiotics susceptible to hydrolysis by these enzymes. An increasing number of ESBLs other then TEM or SHV lineage has recently been described. [3] The presence of ESBL carries tremendous clinical significance. The ESBLs are usually plasmid encoded. Plasmids responsible for ESBL production frequently carry genes encoding resistance to other drug classes like aminoglycosides. Therefore, antibiotic options in the treatment of ESBL- producing organisms are extremely limited. Carbapenems are the treatment of choice for serious infections due to ESBL producing 
organisms, although carbapenem-resistant isolates have recently been reported. ESBLproducing organisms may appear susceptible to some extended spectrum cephalosporins, however treatment with such antibiotics has been associated with high failure rates. ESBLs can be found in a variety of Enterobacteriaceae species, however, the majority of ESBL producing strains are those of Klebsiella pneumoniae, Klebsiella oxytoca and Escherichia coli. Other organisms reported to harbour ESBLs include Enterobacter spp., Salmonella spp., Morganella morganii, Proteus mirabilis, Serratia marcescens and Pseudomonas aeruginosa. However, the frequency of ESBL production in these organisms is low. ${ }^{4,5]}$

Various reports are available regarding the emergence of ESBL producing Escherichia coli strains with an extremely wide spectrum of antibiotic resistance, such as resistance to aminoglycosides, fluoroquinolones, tetracyclines, chloramphenicol and sulfonamides. [6] The $\beta$ lactamase mediated resistance may be overcome by combining $\beta$-lactam antibiotic with $\beta$ lactamase inhibitors, which bind irreversibly to $\beta$-lactamases rendering them inactive, thus sparing the $\beta$-lactam antibiotics. ${ }^{77}$ The Clinical and Laboratory Standards Institute (CLSI) recommends standardized methods for ESBL screening and confirmation. ${ }^{[8]}$ However, their use in microbiology laboratories has been neglected. Delay in the detection and reporting of ESBL production by Gram negative bacteria is associated with prolonged hospital stay and increased morbidity, mortality and health care costs. [9] Institutional microbial sensitivity tests or determination of local patterns of susceptibility are the first steps that are crucial for treatment of ESBL producing bacteria.

AIMS OF THE STUDY: This prospective study, approved by our Institutional Ethics Committee, was conducted with objectives to determine the prevalence of ESBL producing Escherichia coli, to examine their antibiogram profile and to evaluate the association between ESBL production and antibiotic resistance.

MATERIALS AND METHODS: The present study comprised of 286 consecutive, non-repeated isolates of Escherichia coli isolated from various clinical specimens received in the Department of Microbiology, from patients admitted to the wards or attending the outpatient department of Jawaharlal Nehru Medical College, AMU, Aligarh, during the period July 2007 to December 2008. Relevant information, such as in- or out-patient status, hospital stay at the time of sample collection and history of 3rd generation cephalosporin ( $3^{\text {rd }} \mathrm{GC}$ ) use in the preceding two weeks was obtained.

All the samples were processed and identified as per the standard microbiological protocols and procedures. [10] Isolates confirmed as Escherichia coli were studied for their antimicrobial susceptibility pattern and extended spectrum $\beta$-lactamase production. Antimicrobial susceptibility testing was performed using the Kirby-Bauer disk diffusion method with the following set of antibiotics: ampicillin $(10 \mu \mathrm{g})$, piperacillin $(100 \mu \mathrm{g})$, piperacillin/tazobactam (100/10 $\mu \mathrm{g})$, amoxyclav $(20 / 10 \mu \mathrm{g})$, cefoperazone/sulbactam $(75 / 10 \mu \mathrm{g})$, ceftazidime/clavulanate $(30 / 10 \mu \mathrm{g})$, cefoperazone $(75 \mu \mathrm{g})$, cefoxitin $(30 \mu \mathrm{g})$, ceftazidime $(30 \mu \mathrm{g})$, cefotaxime $(30 \mu \mathrm{g})$, ceftriaxone $(30 \mu \mathrm{g})$, cefepime $(30 \mu \mathrm{g})$, aztreonam $(30 \mu \mathrm{g})$, imipenem $(10 \mu \mathrm{g})$, amikacin $(30 \mu \mathrm{g})$, gentamicin $(10 \mu \mathrm{g})$, netilmicin $(30 \mu \mathrm{g})$, ciprofloxacin $(30 \mu \mathrm{g})$, ofloxacin $(5 \mu \mathrm{g})$, and nitrofurantoin $(300 \mu \mathrm{g})$. The antimicrobial disks were obtained from $\mathrm{Hi}$ Media laboratories, Mumbai, India. 
Screening for ESBL production: Ceftazidime and cefotaxime disks were included in the primary panel for screening potential ESBL producers. Isolates with inhibition zone diameter of $\leq 22 \mathrm{~mm}$ for ceftazidime and $\leq 27 \mathrm{~mm}$ for cefotaxime were considered as potential ESBL producers as per the CLSI guidelines and put to confirmatory testing by phenotypic confirmatory disk diffusion tests (PCDDT) (Figure 1). [11,12]

Phenotypic Confirmatory Disk Diffusion Tests (PCDDT): Ceftazidime and cefotaxime disks (30 $\mu$ g each) were used alone and in combination with $10 \mu \mathrm{g}$ of clavulanic acid in the phenotypic confirmatory disk diffusion tests (PCDDT). Individual disks were placed at least $3 \mathrm{~cm}$ centre to centre apart. An increase in zone diameter of either ceftazidime or cefotaxime by $\geq 5 \mathrm{~mm}$ with clavulanic acid versus its diameter when tested alone was considered as ESBL positive (Figure 2). [11,13]

Statistical analysis used: The statistical association between ESBL production and resistance to antibiotics was evaluated using the Chi-square $\left(\chi^{2}\right)$ test.

RESULTS: In the present work, 286 isolates of Escherichia coli were included (107 from pus, 89 from urine, 33 from blood, 15 from stool and throat swab each, 12 from cervical swab, 7 from sputum, 5 from CSF and 3 from conjunctival swab).

Of the total isolates screened for ESBL production, 65.03\% $(n=186)$ were detected to be positive using either ceftazidime or cefotaxime. In the screen positives, 91.94\% $(n=171)$ were ESBL producers by PCDDT method. The overall prevalence of ESBL producing Escherichia coli was $59.79 \%(n=171 / 286)$ with $87.72 \%$ obtained from in-patients and $12.28 \%$ from outpatients. $70.76 \%$ cases had hospital stay duration of more than a week and $89.47 \%$ had a history of prior use of $3^{\text {rd }}$ generation cephalosporin in the preceding two weeks. The differences were statistically significant from non-producers as depicted in Figures 3, 4 and 5 respectively. Majority of ESBL producing Escherichia coli were recovered from stool (73.33\%), followed by pus (62.62\%), urine (61.80\%) and blood (60.61\%) (Figure 6).

The antibiogram of Escherichia coli isolates was determined against a pre-determined panel of antimicrobial agents. The microorganism revealed no resistance to imipenem, while the highest rate of resistance was detected against cefoperazone, amoxicillin/clavulanic acid, ampicillin, cefotaxime, aztreonam, cefoxitin, piperacillin, ceftazidime and ceftriaxone (Table 1).

Drugs that showed statistical correlation as regards percentage of resistant isolates and ESBL production were ceftriaxone $(\mathrm{p}<0.05)$, cefotaxime $(\mathrm{p}<0.001)$, aztreonam $(\mathrm{p}<0.05)$, gentamicin $(\mathrm{p}<0.05)$, ceftazidime $(\mathrm{p}<0.05)$ and nitrofurantoin $(\mathrm{p}<0.05)$. The remaining antibiotics revealed no significant association (Table 2). Although there was no significant statistical difference between resistance to most of the tested drugs and ESBL production, the strains producing ESBL showed a higher resistance to all the used antibiotics except for ofloxacin (Table 2).

DISCUSSION: During the past decade, ESBL producing Gram negative bacilli, especially Escherichia coli, have emerged as serious pathogens both in hospital and community acquired infections worldwide.

In our study, the highest susceptibility of E. coli isolates was found to imipenem (100\%) followed by piperacillin/tazobactam (87.41\%), cefoperazone/sulbactam (76.92\%) and ceftazidime/clavulanic acid (66.43\%). Studies by Akram et al. (2007) and Babypadmini et al. 
(2004) ${ }^{[14,15]}$ on urinary isolates of Escherichia coli also showed the susceptibility of Escherichia coli to imipenem as $100 \%$. Menon et al. (2006) [16], in their study, also reported almost similar results of susceptibility for to imipenem, piperacillin/tazobactam, cefoperazone/sulbactam and ceftazidime/clavulanic acid with slight variations from above quoted values.

In Escherichia coli, high resistance was observed with $3^{\text {rd }}$ generation cephalosporins [cefotaxime (68.53\%), ceftazidime (64.34\%) and ceftriaxone (61.54\%)], cephamycin (cefoxitin) (68.53\%), monobactam (aztreonam) (68.53\%), piperacillin (66.43\%), cefoperazone $(72.03 \%)$, cefepime (65.73\%), amoxicillin/clavulanic acid (71.68\%) and gentamicin (61.54\%). Akram et al. (2007), Babypadmini et al. (2004) and Menon et al. (2006) [14, 15, 16] also documented almost similar results.

The overall prevalence of ESBL in Escherichia coli in the current study was 59.79\%. Previous studies from India have reported prevalence of ESBL producers to be $6.6 \%-68 \%$. [17, 18, 19, 20] The occurrence of ESBLs varied from one locality to another, which may be due to infection control practice among different regions or to the differences in the uses of new extended spectrum antimicrobial agents.

In several western studies, prevalence of ESBLs in Escherichia coli was less than to our study. The higher prevalence compared to western countries can be explained by the fact that western countries have strict infection control policies and practices, efficient and effective antibiotic audit systems, shorter average hospital stays, better nursing barriers and other important health care measures that are known to substantially decrease the chances of acquisition and spread of ESBL strains. The uncontrolled use of $3^{\text {rd }}$ generation cephalosporins at our hospital could be a leading contributory factor to the high ESBL prevalence observed in this study.

The differences in the in- and out-patient distribution between ESBL producers and nonproducers in the present study were statistically significant. These results point to the fact that ESBLs are largely a problem of hospitalized patients who share numerous risk factors.

There was a statistically significant difference in the hospital stays of patients infected with ESBL producing and non producing Escherichia coli. Prolonged hospital stay as such is a very important risk factor for acquisition and transmission of an ESBL producing strain. The present study also revealed a statistically significant difference between the prior use of $3^{\text {rd }}$ generation cephalosporins and subsequent infection with an ESBL producing or non-producing Escherichia coli. The increasing use of broad spectrum cephalosporins has become one of the major factors responsible for the high rate of selection of ESBL producing microorganism. [21]

The ESBL producers showed very high resistance to $\beta$-lactam antimicrobials compared to non- producers that appeared uniformly susceptible. The differences were statistically significant. Resistance conferred by ESBL producing Escherichia coli to ceftazidime, cefotaxime and ceftriaxone was $91.25 \%, 93.24 \%$ and $92.25 \%$ respectively.

The associated co-resistance to non- $\beta$-lactams, gentamicin, ciprofloxacin and nitrofurantoin by ESBL producers was $83.55 \%, 42.1 \%$ and $68.4 \%$ respectively. Non-producers were susceptible to all these drugs. The differences were statistically significant in our study. Similar resistance patterns have been reported from other parts of India. [15, 22]

Our study results well support the fact that ESBL producers not only confer high levels of resistance to $3^{\text {rd }}$ generation cephalosporins but also to non $\beta$-lactams like aminoglycosides and quinolones. 
CONCLUSIONS: This study highlights the emergence of ESBL producing strains of Escherichia coli which are endowed with extremely wide spectrum of antibiotic resistance including resistance to penicillins, cephalosporins, aminoglycosides, and fluoroquinolones. This has lead to significant implications in the management of patients. Periodic review of ESBL producing strains as regards their prevalence in an area as well as their antibiotic susceptibility profiles is mandatory to understand the ever growing magnitude of this problem. Advanced drug resistance surveillance and determination of molecular characteristics of ESBL isolates are also required so as to help formulate antibiotic prescription policies, and ensure appropriate and judicious use of the available antimicrobial drugs.

\section{REFERENCES:}

1. Livermore DM. Beta-lactamases in laboratory and clinical resistance. Clin Microbiol Rev 1995; 8: 557-584.

2. Bush K, Jacoby GA, Medeiros AA. A functional classification scheme for beta-lactamases and its correlation with molecular structure. Antimicrob Agents Chemother 1995; 39: 12111233.

3. Paterson DL, Bonomo RA. ESBL: a clinical update. Clinical Microbiology Reviews 2005; 18 : 657-686.

4. Chaudhray U, Aggarwal R. Extended spectrum- $\beta$-lactamases (ESBL): An emerging threat to clinical therapeutics. IJMM 2004; 22:75-80.

5. Nathisuwan S, Burges DS, Lewis II JS. ESBLs Epidemiology, detection and treatment. Pharmacotherapy 2001; 21: 920-928.

6. Kaye KS, Engemann JJ, Fraimow HS, Abrutyn E. Pathogens resistant to antimicrobial agents: Epidimiology, molecular mechanisms and clinical management. Infect Dis Clin N Am 2004; 18: 467-511.

7. Monhanty S, Singhal R, Sood S. Comparative in vitro activity of beta lactam / beta lactamases inhibitor combinations against gram negative bacteria. Indian J Med Res 2005; 122: 425428.

8. National Committee for Clinical Laboratory Standards. Approved standards M-100- S10. Performance standards for antimicrobial susceptibility testing. Wayne (USA). National Committee for Clinical Laboratory Standards 2000.

9. Kollef MH. The importance of appropriate initial antibiotic therapy for hospital acquired infection. Am J Med 2003; 115: 582-584.

10. Crichton PB. Enterobacteriaceae: Escherichia, Klebsiella, Proteus and other genera. In: Collee JG, Fraser AG, Marmion BP, Simmons A, editors. Mackie and McCartney Practical Medical Microbiology. 14th ed. Edinburgh: Churchill Livingstone; 2006; p.368-370.

11. Livermore DM, Woodford N. Laboratory detection and reporting of bacteria with extended spectrum $\beta$-lactamases. Antibiotic resistance monitoring and reference laboratory, specialist and reference Microbiology division, Health Protection Agency, Colindale, London, June 2004.

12. NCCLS performance standards for antimicrobial susceptibility testing; Eighth informational supplement. NCCLS document M100- S8. NCCLS, Wayne, PA 1998.

13. Parasakthi N, Ariffin H. Consensus guidelines for the management of infections by ESBL producing bacteria, Ministry of Health, Malaysia. Academy of Medicine of Malaysia. Malaysian Society of Infectious Disease and Chemotherapy 2001. 
14. Akram M, Shahid M, Khan AU. Etiology and antibiotic resistance patterns of communityacquired urinary tract infections in J N M C Hospital Aligarh, India. Annals of Clinical Microbiology and Antimicrobials 2007; 6: 4-10.

15. Babypadmini S, Appalaraju B. Extended spectrum beta lactamases in urinary isolates of E.coli \& K.pneumoniae-prevalence and susceptibility pattern in a tertiary care hospital. Indian J Med Microbiol 2004; 22: 172-174.

16. Menon T, Bindu D, Kumar CP, Nalini S, Thirunarayan MA. Comparison of double disk and three dimensional methods to screen for ESBL producers in a tertiary care hospital. Indian J Med Microbiol 2006; 24: 117-120.

17. Jain A, Roy I, Gupta MK, Kumar M, Agarwal SK. Prevalence of extended-spectrum betalactamase-producing Gram negative bacteria in septicaemic neonates in a tertiary care hospital. J Med Microbiol 2003; 52: 421-425.

18. Mathur P, Kapil A, Das B, Dhawan B. Prevalence of extended spectrum beta lactamase producing Gram negative bacteria in a tertiary care hospital. Indian J Med Res 2002; 115: 153-157.

19. Datta P, Thakur A, Mishra B, Gupta V. Prevalence of clinical strains resistant to various $\beta$ lactamases in a tertiary care hospital in India. Jpn J Infect Dis 2004; 57: 146-149.

20. Tankhiwale SS, Jalgaonkar SV, Ahamad S, Hassani U. Evaluation of extended spectrum beta lactamase in urinary isolates. Indian J Med Res 2004; 120: 553-556.

21. Pallucha A, Mikieweiz B, Hryniewiez W, Griadkowshki M. Concurrent outbreaks of ESBL producing organisms of the family Enterobacteriaceae in a Warsaw hospital. J Antimicrob Chemother. 1999; 44: 489-499

22. Ananthakrishnan AN, Kanungo R, Kumar A, Badrinath S. Detection of ESBL producers among surgical wound infections and burns patients in JIPMER. Indian J Med Microbiol 2000; 18: 160-165. 
Table 1: Antimicrobial susceptibility pattern in Escherichia coli isolates

\begin{tabular}{ccc}
\hline \multirow{2}{*}{ Antibiotics } & \multicolumn{2}{c}{ Number of isolates (n=286) } \\
\cline { 2 - 3 } & Sensitive & Resistant \\
\hline Ampicillin & $86(30.07)$ & $200(69.93)$ \\
Piperacillin & $96(33.57)$ & $190(66.43)$ \\
Piperacillin/Tazobactam & $250(87.41)$ & $36(12.59)$ \\
Amoxycillin/Clavulanic acid & $81(28.32)$ & $205(71.68)$ \\
Cefoperazone/Sulbactam & $220(76.92)$ & $66(23.08)$ \\
Ceftazidime/Clavulanic acid & $190(66.43)$ & $96(33.57)$ \\
Cefoperazone & $80(27.97)$ & $206(72.03)$ \\
Cefoxitin & $90(31.47)$ & $196(68.53)$ \\
Ceftazidime & $102(35.66)$ & $184(64.34)$ \\
Cefotaxime & $90(31.47)$ & $196(68.53)$ \\
Ceftriaxone & $110(38.46)$ & $176(61.54)$ \\
Cefepime & $101(35.31)$ & $188(65.73)$ \\
Aztreonam & $90(31.47)$ & $196(68.53)$ \\
Imipenem & $286(100)$ & $0(0.00)$ \\
Amikacin & $199(69.58)$ & $87(30.42)$ \\
Gentamicin & $110(38.46)$ & $176(61.54)$ \\
Netilmicin & $159(55.59)$ & $127(44.41)$ \\
Ciprofloxacin & $135(47.20)$ & $151(52.80)$ \\
Ofloxacin & $155(54.20)$ & $131(45.80)$ \\
\hline
\end{tabular}

Figures in parentheses indicate percentage of isolates 
Table 2: Percentage of antimicrobial resistance and $P$ value results in ESBL producing and non-ESBL producing Escherichia coli $(n=286)$

\begin{tabular}{|c|c|c|c|c|}
\hline \multirow[b]{2}{*}{ Antibiotics } & \multicolumn{2}{|c|}{ Escherichia coli } & \multirow[b]{2}{*}{$\chi^{2}$ test results } & \multirow[b]{2}{*}{$P$ value } \\
\hline & $\begin{array}{c}\% \text { of } \\
\text { resistance in } \\
\text { ESBLs } \\
\text { producers } \\
(n=186)\end{array}$ & $\begin{array}{c}\% \text { of } \\
\text { resistance in } \\
\text { non-ESBLs } \\
\text { producers } \\
(n=100)\end{array}$ & & \\
\hline Ampicillin & 100 & 95.2 & 0.8211 & $<0.5$ \\
\hline Piperacillin & 99.7 & 82.4 & 1.804 & $<0.5$ \\
\hline Nitrofurantoin & 68.4 & 40.2 & 40.0367 & $<0.05$ \\
\hline Cefoperazone & 100 & 98.9 & 0.8411 & $<0.5$ \\
\hline Ceftazidime & 91.25 & 67.8 & 4.325 & $<0.05$ \\
\hline Cefotaxime & 93.24 & 71.44 & 6.0381 & $<0.001$ \\
\hline Ceftriaxone & 92.25 & 74.1 & 4.012 & $<0.05$ \\
\hline Cefepime & 72.61 & 44.04 & 3.894 & $<0.1$ \\
\hline Aztreonam & 100 & 77.30 & 3.8789 & $<0.05$ \\
\hline Amikacin & 42.1 & 24.7 & 0.1607 & $<0.5$ \\
\hline Gentamicin & 83.55 & 57.2 & 3.982 & $<0.05$ \\
\hline Ciprofloxacin & 42.1 & 37.1 & 0.022 & $<0.5$ \\
\hline Ofloxacin & 21.1 & 32.9 & 0.5735 & $<0.5$ \\
\hline
\end{tabular}

Figure 1: Screening of ESBL by Kirby $\quad$ Figure 2: Phenotypic confirmatory Double Disk Bauer Disk Diffusion method. Diffusion Test (PCDDT) for the detection of ESBL production.
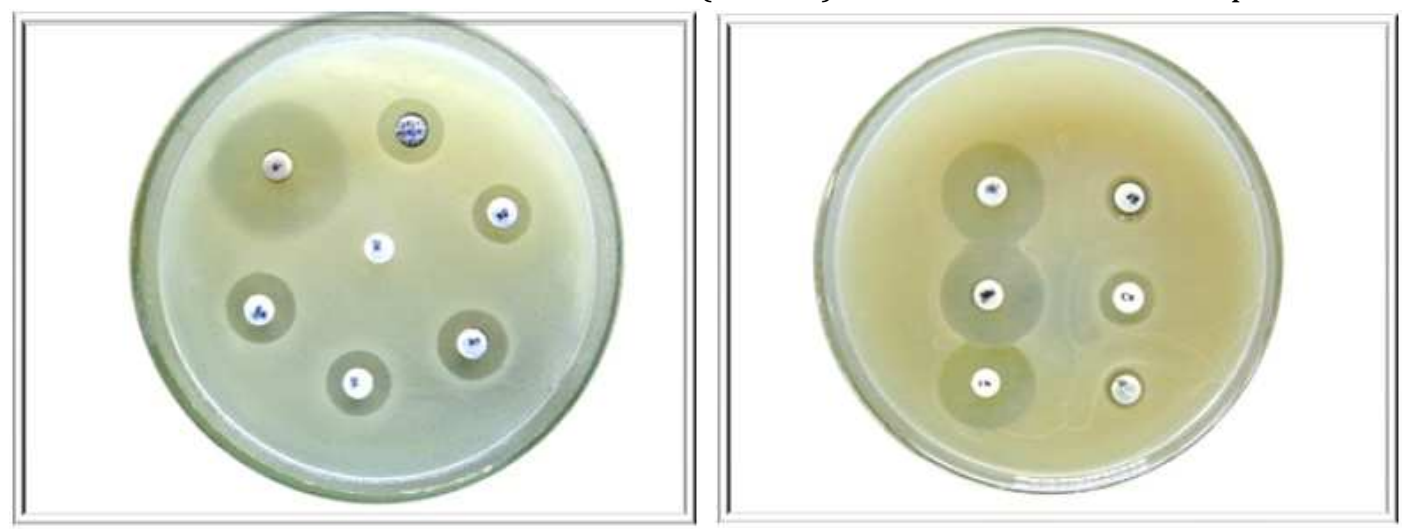
Figure 3: Distribution pattern of ESBL producers \& non-producers in out-patients and inpatients.

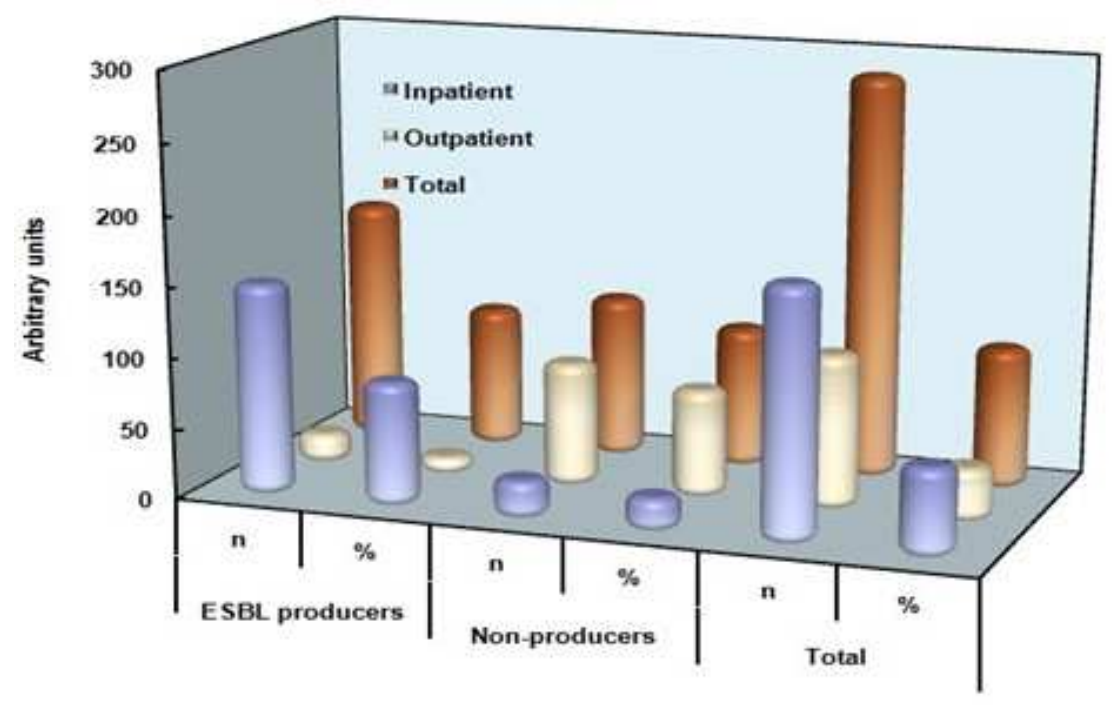

$P$ value $<0.004$

Figure 4: Duration of hospital stay of patients infected with ESBL producing and non-producing Escherichia coli.

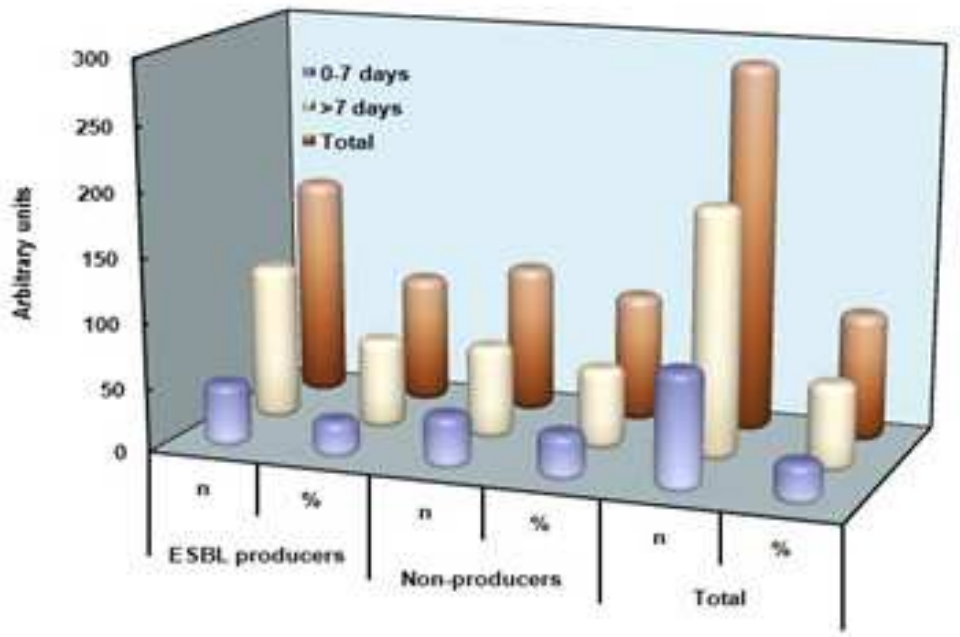


Figure 5: History of prior $3^{\text {rd }}$ generation cephalosporin consumption in patients infected with ESBL producing and non-producing Escherichia coli.

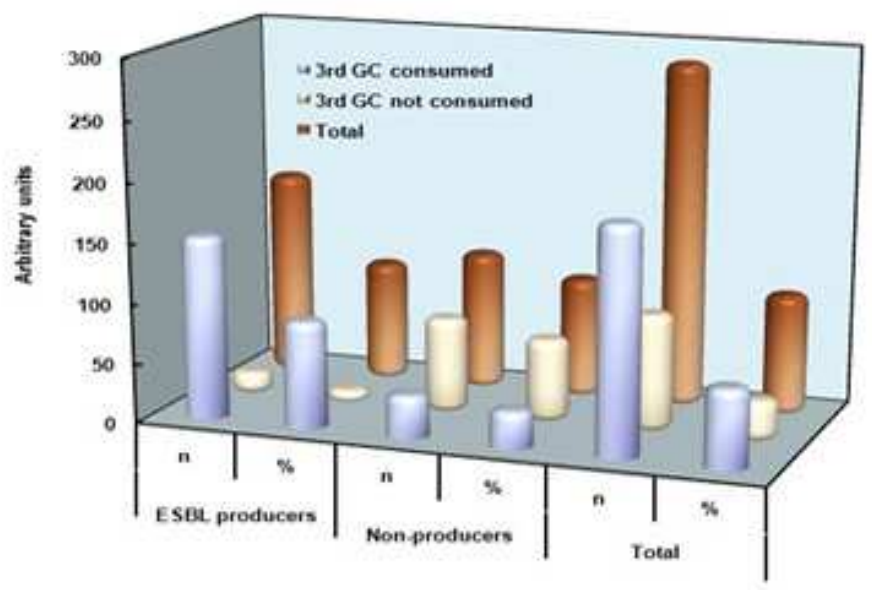

Figure 6: Percentage detection of ESBL in Escherichia coli by PCDDT method in different samples.

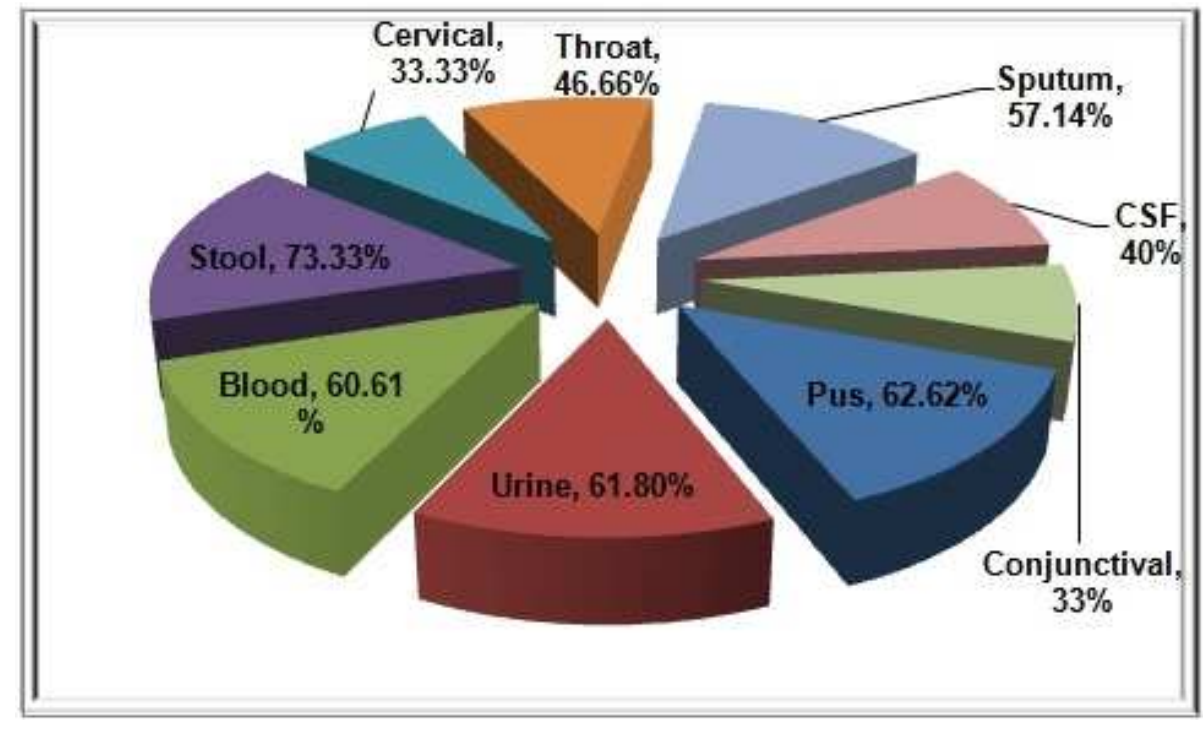

